\title{
中国种堂
}

\section{小角 $\mathrm{X}$ 射线散射技术研究非晶合金回火 过程中结晶-非晶两相系统比内表面}

\author{
孟昭富王显明
}

(吉林大学材料科学研究所, 长春 130023 )

\section{摘 要}

本文应用小角 $\mathrm{X}$ 射线散射技术 (SAXS)，结合广角 $\mathrm{X}$ 射线衍射（WAXD）确定 了 $\mathrm{Cu}_{73} \mathrm{Sn}_{6} \mathrm{Ni}_{6} \mathrm{P}_{15}$ 非晶合金经过 $473 \mathrm{~K}$ 回火后形成的结晶一非晶两相系统的比内表 面. 结果表明这个非晶合金在 $473 \mathrm{~K}$ 回火过程中, 当回火时间从 $5 \mathrm{~min}$ 增加到 $4 \mathrm{~h}$ 时, 两相系统比内表面从 $144 \mathrm{~m}^{2} / \mathrm{cm}^{3}$ 减少到 $96 \mathrm{~m}^{2} / \mathrm{cm}^{3}$. 本文确定出的两相系统比 内表面的误差约为 $7 \%$ 。

关键词: 小角 $\mathrm{X}$ 射线散射，非昆合金,比内表面

\section{一、引 言}

小角 X 射线散射技术 (SAXS) 广泛地用来研究 $1.0 \mathrm{~nm}-200 \mathrm{~nm}$ 尺寸范围的各种微小粒 子. 最近, SAXS 技术已被作者用于确定工程铝合金中析出的相粒子尺寸和形状、非晶合金 中回火生成的晶化相粒子的特征函数 ${ }^{[1,2]}$. 近年来也有一些作者应用 SAXS 技术研究非晶合 金的晶化和结构弛豫过程 ${ }^{[3,4]}$, 但到目前为止尚没有人研究结晶-非晶两相系统的比内表面. 本 文应用 SAXS 技术并结合运用广角X射线衍射 (WAXD) 研究了 $\mathrm{Cu}_{73} \mathrm{Sn}_{6} \mathrm{Ni}_{6} \mathrm{P}_{15}$ 非晶合金在 $473 \mathrm{~K}$ 回火过程中结晶-非晶两相系统比内表面的改变.

两相系统比内表面是一个重要的物理量, 在时效硬化合金中, 它同材料的力学性质及抗应 力腐蚀能力有关; 在载体催化剂中, 它同催化剂的物理化学性质有关; 在非晶合金回火后生成 的部分晶化结构的两相系统中，它同非晶合金的脆化及物理性质的改变有关. 因此研究确定 两相系统比内表面的方法有一定的理论和应用方面的意义.

\section{二、实验}

试样采用 $\mathrm{Cu}_{73} \mathrm{Sn}_{6} \mathrm{Ni}_{6} \mathrm{P}_{15}$ 非晶合金条带，小角 $\mathrm{X}$ 射线散射试样的尺寸为 $35 \times 5 \times 0.03$ $\mathrm{mm}^{3}$ 。试样在硅油炉中经过 $473 \mathrm{~K}$ 不同的时间回火处理, 使其达到不同的结晶程度。采用 $\mathrm{D} / \mathrm{max}-\gamma \mathrm{A}$ 型 $12 \mathrm{~kW}$ 旋转阳极 $\mathrm{X}$ 射线衍射仪. 小角 $\mathrm{X}$ 射线散射使用三长狭缝准直系统，第 一狭缝宽 $0.04 \mathrm{~mm}$, 第二狭缝宽为 $0.03 \mathrm{~mm}$, 第三狭㖓宽为 $0.05 \mathrm{~mm}$, 计数管接受狭㖓宽为 
$0.1 \mathrm{~mm}$. 采用 $\mathrm{CuK} \alpha$ 辐射, $\mathrm{Ni}$ 滤片,管压 $50 \mathrm{kV}$, 管流 $150 \mathrm{~mA}$. 采用步进扫描,步宽 $0.02^{\circ}$, 每步时间 $40 \mathrm{~s}$, 测量的角度范围为 $0.1^{\circ}-2^{\circ}$. 散射强度扣除背底散射, 得到长狭㖓准直的实 验散射强度 $J(\varepsilon)$ 或 $J(s), J(h)$, 这里 $\varepsilon$ 为散射角, $s=2 \sin (\varepsilon / 2) / \lambda, h=2 \pi s, \lambda$ 为 X 射 线波长. 本文中的计算工作是在 IBM 机上进行使用 BASIC 程序. 广角 X射线衍射实验是 在同一台衍射仪上进行, 使用 $\mathrm{Mo} \mathrm{K} \alpha$ 辐射, 石墨单色器.

\section{三、实 验结 果}

图 1、图 2 分别给出了实验得出的 $J(\varepsilon)-\varepsilon$ 和 $\ln J(h)-h^{2}$ 曲线. 由图 2 曲线低角部分的 斜率可求得结晶粒子的迴转半径 $R_{G}{ }^{\left[{ }^{[9]}\right.}$ :

$$
R_{G}=\sqrt{-3 \alpha}
$$

$\alpha$ 为 $\ln J(h)-h^{2}$ 曲线低角部分直线斜率。

由图 2 和 (1) 式求出 $\mathrm{Cu}_{73} \mathrm{Sn}_{6} \mathrm{Ni}_{6} \mathrm{P}_{15}$ 非晶合金在 $473 \mathrm{~K}$ 经过不同的时间回火处理后的结 晶粒子的迴转半径 $R_{G}$ 列于表 1 .

两相系系统比内表面 $s_{p}$ 由下式决定 ${ }^{[0]}$

$$
s_{p}=c(1-c) \lim h^{j} J(h) / \int_{0}^{\infty} h J(h) d h,
$$

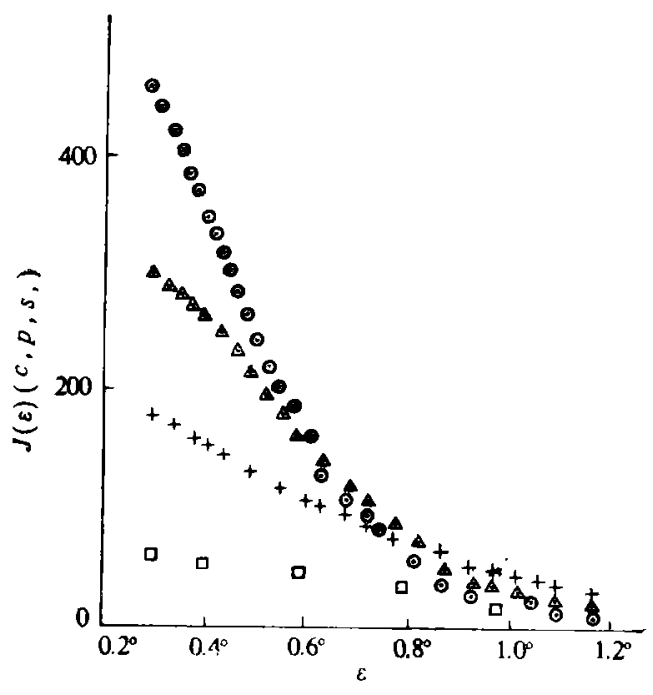

图 $1 J(\varepsilon)-\varepsilon$ 曲线

$(0-473 \mathrm{~K}, 4 \mathrm{~h}$ 回火试样; $\Delta-473 \mathrm{~K}, 30 \mathrm{~min}$

回火试样; $+-473 \mathrm{~K}, 5 \mathrm{~min}$ 回火试样; 口- 未回火非晶试样)

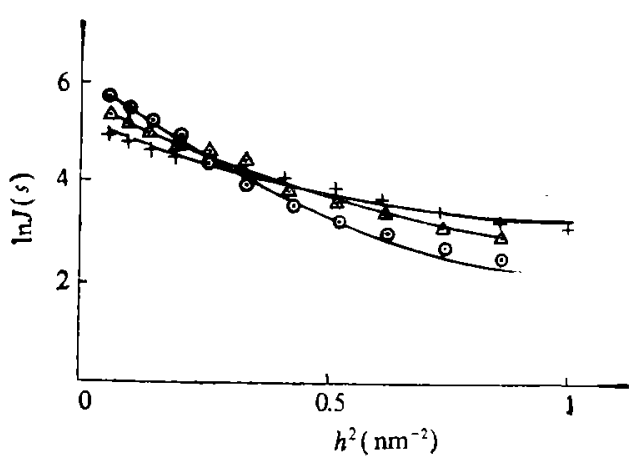

图 $2 \ln J(h)-h^{2}$ 曲线

$(\mathrm{O}-473 \mathrm{~K}, 4 \mathrm{~h}$ 回火试样; $\Delta-473 \mathrm{~K}, 30 \mathrm{~min}$

回火试样; +— $473 \mathrm{~K}, 5 \mathrm{~min}$ 回火试样)

这里 $c$ 是结晶粒子的体积分数, $\lim h^{3} J(h)$ 是 $h$ 趋于大值时, $h^{3} J(h)$ 的极限值. 令

$$
K_{p}^{\prime}=\lim s^{3} J(s)=1 /(2 \pi)^{3} \lim h^{3} J(h),
$$

$K_{p}^{\prime}$ 为一常数值,

$$
\widetilde{Q}_{s}=\int_{0}^{\infty} s J(s) d s=1 /(2 \pi)^{2} \int_{0}^{\infty} h J(h) d h,
$$


则有

$$
s_{p}=8 \pi c(1-c) K_{p}^{\prime} / \widetilde{Q} .
$$

为了精确地计算 $\widetilde{Q}$, , 可将 (4) 式改写为

$$
\widetilde{Q} s=\int_{0}^{\infty} s J(s) d s=\int_{0}^{s_{1}} s J(s) d s+\int_{s_{1}}^{s_{2}} s J(s) d s+\int_{s_{2}}^{\infty} s J(s) d s \text {. }
$$

在 (6) 式中, 只有在积分区间 $s_{1}-s_{2}$ 内, $J(s)$ 可以直接被测出实验值; 在 (6) 式中的第 一个积分区间 0-s 内, 由于 $\varepsilon$ 值太小, 实验不能直接测出此区间的 $J(s)$, 但可由 $\ln J(h)-h^{2}$ 曲线在低角部分成为直线而外推出此区间的 $J(s)$ 值来. 在 (6) 式中第三个积分区间 $s_{2}-\infty$ 内, 是具有大的 $s$ 值的区间, 它是测量角度范围之外的区间，因而在此区间也不可能获得实测 的 $J(h)$ 值, 但是当粒子具有明锐的界面的情况下,在此区间 (3) 式成立 ${ }^{[6]}$,则利用 (3)式有

$$
\int_{s_{2}}^{\infty} s J(s) d s=\int_{s_{2}}^{\infty} \frac{s_{2}^{3} J\left(s_{2}\right)}{s^{2}} d s=s_{2}^{2} J\left(s_{2}\right) \text {. }
$$

使用上面的方法, 利用 (6), (7) 式可获得精确的 $\widetilde{Q}$ 。值. 表 1 中列出了 $\widetilde{Q}$ 值和 $K_{p}^{\prime}$ 值.

图 3 为 $s^{3} J(s)-s$ 曲线, 从它可见, 当 $s$ 趋于大值时, $s^{3} J(s)$ 趋于常数, 从而说明了使用 (7) 式的合理性,也表明结晶粒子和非晶基体间具有明锐的相间界面.

为了使用 (5) 式计算两相系统的比内表面, 必须计算出结晶粒子的体积分数 $c$. 在本文 中利用广角 $\mathrm{X}$ 射线衍射 (WAXD) 确定 $c$ 值. 在晶化初期的非晶合金的 $\mathrm{X}$ 射线广角衍射花样 上, 除原来的非晶漫散峰外, 又出现结晶相的尖锐的衍射峰. 由于非晶峰十分漫散, 扩展到 $2 \theta$ 为十几度的角度范围, 在一般情况下, 结晶相的尖锐衍射峰, 重迭在非晶漫散峰之上, 但两 者的顶峰不在相同的角度上, 正如图 (b) 所指出的那样.

为了确定结晶粒子的体积分数 $c$, 仅需要讨论第一个非晶峰和重迭在它上面的结晶峰. 我们已经指出，第一个非晶散射峰在 $2 \theta_{1}-2 \theta_{2}$ 角度范围内的总积分强度为 ${ }^{[7]}$

$$
\begin{aligned}
& I_{a}^{\prime}=k_{a} \nu_{a} \int_{2 \theta_{1}}^{2 \theta_{2}} I^{\mathrm{Coh}}(h) e^{-2 M} A(\theta) \varphi(\theta) d(2 \theta), \\
& I^{\mathrm{coh}}(h)=\left\langle f^{2}\right\rangle+\langle f\rangle^{2} \int_{0}^{\infty} 4 \pi r^{2}[\rho(r)-\rho(0)] \frac{\sin (h r)}{h r} d r .
\end{aligned}
$$

${ }^{c 0 h}(h)$ 是相一散射强度. $k_{a}$ 是依赖于实验条件的常数， $v_{a}$ 是 X 射线照射的试样内非晶相的 体积, $e^{-2 M}$ 是 Debye-Waller 因子, 它依赖于 $\theta$, 并与材料的种类有关, $\theta=\varepsilon / 2, A(\theta)$ 为 吸收因子, $\varphi^{\prime}(\theta)$ 为角因子, $f$ 为原子散射因子, $\rho(r)$ 为 $r$ 处的原子密度, $\rho_{0}$ 为平均原子密 度.

在 (8) 式中我们仅考虑相干散射强度. 我们已指出 ${ }^{[7]}$, 在所考虑的散射峰角度范围内, 非 相斗散的强度相对于相干散射强度弱得多, 并且随角度改变很小, 可以作为背底来处理 ${ }^{[7]}$. 在 (9) 式中 $\left[\rho(r)-f_{0}\right]$ 论顿于非晶部分的原子结构, 我们已指出 ${ }^{[7]}$, 它在晶化初期的试样和在 完全未晶化的非昆试样上，几䓅没有区别. 将 (8) 式中的积分部分表示为 $Q_{a}$, 则 $Q_{a}$ 。在部分 晶化前后几乎相同,于是有

$$
I_{a}^{\prime}=k_{a} v_{a} Q_{a}=k_{a} c_{a} v Q_{a},
$$

这里 $c_{a}$ 为非晶相的体积分数, $"$ 次 X 射线照射的试样体积.

未回火的非晶试样,处于完全等状态, 在实验条件不变的情况下, 第一个非晶峰在相同 


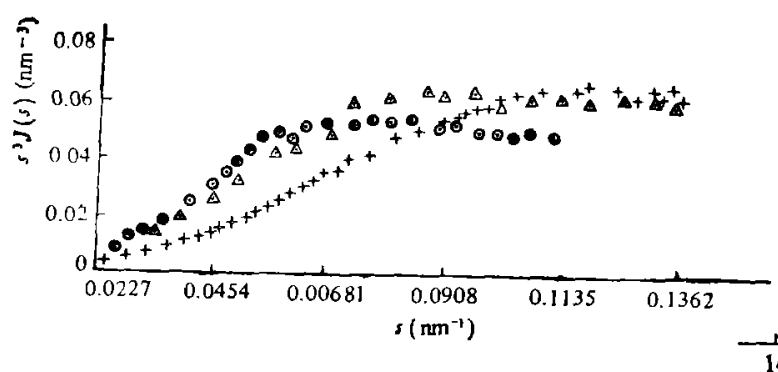

图 $3 s^{3} J(s)-s$ 关系图

( o-4 $473 \mathrm{~K}$, 4h 回火试样; $\triangle-473 \mathrm{~K}, 30 \mathrm{~min}$ 回火试样; +

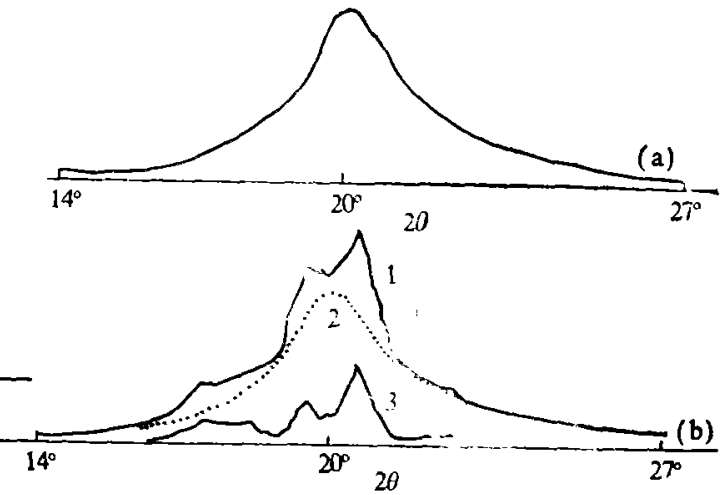

图 4 （a）末回火的完全非昆状态试样的䈍 一个散射峰；(b) $473 \mathrm{~K} ， 4 \mathrm{~h}$ 回火试样在相 同角度范围的衍射花样

(曲线 1 为非晶峰和结晶峰的重迭行射花样; 嚄有点线的 曲线 2 为分开后的非晶峰; 曲线 3 为分开后的结: 暑峰)

的角度范围内的总积分强度为

$$
I_{a}=k_{a} v Q_{a}
$$

由（10),(11) 式有

$$
c_{a}=\frac{I_{a}^{\prime}}{I_{a}}
$$

并且

$$
c_{a}+c=1,
$$

由 (12), (13) 式可以求出结晶愔子的体积分数 $c$.

为了确定 $c$, 关键的步骤是分开重迭在一起的结晶峰和非晶峰. 图 4(a) 为完全非晶状 态试样的散射花样, 图 4 (b) 为 $473 \mathrm{~K}, 4 \mathrm{~h}$ 回火后部分结晶试样在相同角度范围内的 X 射线 衍射花样, 从它可看到结晶相衍射峰重迭在非晶相的漫散的散射峰之上. 采用我们在参考文 献 [7] 中描述的方法分开结晶峰和非晶峰. 以图 4(a) 的完全非晶状态试样的漫散峰为标 准, 计算出它在各个角度上的散射强度比例, 则图 4(b) 中的非晶漫散峰在各个角度上的散射 强度应该有与此相同的比例。则从图 4(b) 中某一个远离结晶峰的角度上的非晶峰散射强 度, 按着上述的图 4(a) 各角度上散射强度比例, 求出图 4(b) 中的非晶散射峰线形, 如图 4 (b) 中的曲线 2 所指出的. 而图 4 (b) 中的曲线 1 是非晶峰和结晶峰的重迭线形; 曲线 3 是曲线 1 减去曲线 2 得到的结晶峰线形。计算出图 4(a) 在 $2 \theta$ 角范围为 $14^{\circ}-27^{\circ}$ 的总积

表 $1 \mathrm{Cu}_{73} \mathrm{~S}_{\mathrm{n}_{6}} \mathrm{Ni}_{6} \mathrm{P}_{15}$ 非晶合金 $473 \mathrm{~K}$ 回火不同的时间的 $c, K_{p}^{\prime}, \tilde{Q}_{s}, R_{G}$ 和 $s_{p}$ 的值

\begin{tabular}{c|c|c|c|c|c}
\hline 回火条件 & $c$ & $\begin{array}{c}K_{p}^{\prime} \\
\left(\mathrm{n} \mathrm{m}^{-5}\right)\end{array}$ & $\begin{array}{c}\tilde{Q} \\
\left(\mathrm{~nm}^{-2}\right)\end{array}$ & $\begin{array}{c}s_{p} \\
\left(\mathrm{~m}^{2} / \mathrm{cm}^{3}\right)\end{array}$ & $\begin{array}{c}R_{G} \\
(\mathrm{~nm})\end{array}$ \\
\hline $473 \mathrm{~K}, 5 \mathrm{~min}$ & 0.130 & $6.4 \times 10^{-2}$ & 1.26 & 144 & 2.74 \\
\hline $473 \mathrm{~K}, 30 \mathrm{~min}$ & 0.134 & $6.2 \times 10^{-1}$ & 1.55 & 117 & 3.67 \\
\hline $473 \mathrm{~K}, 4 \mathrm{~h}$ & 0.146 & $5.0 \times 10^{-2}$ & 1.63 & 96 & 5.48 \\
\hline
\end{tabular}


分强度 $I_{a}$, 即曲线下的面积, 而图 4 (b) 中曲线 2 下面的面积为 $I_{a}^{\prime}$, 从而由 (12), (13) 式 计算出结晶粒子的体积分数 $c$, 并列于表 1 中. 由 $c, K_{p}^{\prime}$ 和 $\widetilde{Q}_{s}$ 的俻利用 (5) 式计算出结 晶一非晶两相系统的比内表面 $s_{p}$ 的值幸列入表 1 中.

\section{四、讨 论}

在本文中我们首次使用小角 $\mathrm{X}$ 射线散射技术并且结合广角 $\mathrm{X}$ 射线衍射确定了结晶-非晶 两相系统比内表面. 本文确定两相系统比内表面的方法有广泛的应用前景，例如可应用于时 效硬化合金的析出相一基体两相系统的比内表面的研究，它将对进一步了解材料的力学性能和 抗应力腐蚀性能与时效处理条件间的关系的机理提供一种途径。在非晶合金晶化和结构弛豫 过程中,结晶-非晶两相系统比内表面发生改变。进一步研究合金脆化和热处理条件及比内表 面三者间的关系，将有助于了解非晶合金回火脆化过程的机理. 两相系统比内表面的测定在 催化剂系统中也有广泛地应用 ${ }^{[8]}$.

从本文表 1 可以看到在 $\mathrm{Cu}_{73} \mathrm{Sn}_{6} \mathrm{Ni}_{6} \mathrm{P}_{15}$ 非晶合金的 $473 \mathrm{~K}$ 回火过程中，随着回火时间的 增加, 结晶粒子的迴转半径增加, 结晶粒子的体积分数改变很小, 仅略有增加, 而两相系统的比 内表面大幅度地减少。这表明了结晶粒子的长大过程.

本文使用广角 $\mathrm{X}$ 射线衍射确定了结晶粒子的体积分数 $c$. 在一些情况也可以用 DSC 热 分析仪来确定非晶合金在等温回火过程中生成的结晶相的体积分数. Scott 采用等温退火的 DSC 曲线研究了 2826 非晶合金在不同温度下结晶粒子的体积分数随退火时间的改变，并确 定了晶化动力学参数，他指出 DSC 法仅在有单一放热峰的非晶合金中可以得到正确的结晶 相体积分数的测定结果，而对于有多个放热峰的非晶合金不适宜用 DSC 法确定结晶相的体 积分数 ${ }^{[0]}$. 用广角 $\mathrm{X}$ 射线衍射法不存在上述问题.

在本实验中结晶相体积分数 $c$ 的误差为 $\Delta c= \pm 0.002, K_{p}^{\prime}$ 的误差为 $\Delta K_{p}^{\prime}= \pm 0.001$ $\mathrm{nm}^{-3}, \widetilde{Q}$, 的误差为 $\Delta \widetilde{Q} s= \pm 0.03 \mathrm{~nm}^{-2}$. 将 (5) 式写为

$s_{p}=8 \pi c(1-c) / K_{p}^{\prime} \widetilde{Q}_{s}=f\left(c, K_{p}^{\prime}, \widetilde{Q}_{s}\right)$, 则有

$$
\Delta s_{p}=\left|\frac{\partial f}{\partial c}\right| \Delta c+\left|\frac{\partial f}{\partial K_{p}^{\prime}}\right| \Delta K_{p}^{\prime}+\left|\frac{\partial f}{\partial \widetilde{Q}_{s}}\right| \Delta \widetilde{Q}_{s} .
$$

代人表 1 中的 $473 \mathrm{~K}$ 回火 $5 \mathrm{~min}$ 试样的 $c, K_{p}^{\prime}$ ， $\widetilde{Q}_{s}$ 数值和上面的 $\Delta c, \Delta K_{p}^{\prime}, \Delta \widetilde{Q}$ s的数值， 得到 $\Delta s_{p} \doteq \pm 0.01 \mathrm{~nm}^{-1}= \pm 10 \mathrm{~m}^{2} / \mathrm{cm}^{3}$, 则比内表面 $s_{p}$ 的误差约为 $7 \%$, 对其他回火条件的 试样得到与此相同的值.

\section{参考文献}

[1] 孟昭富,科学通报, 33(1988),22: 1723-1726.

[2] 孟昭富,吉林大学自然科学学报, 1989,3: 2185-2190.

[3] Wu, T.W. \& Speapen,, F., Acta Metall., 33(1985), 2185-2190.

[4] Chou, C.P. \& Turnbull, D.J., Non-Cryst. Solids. 17(1975), 168.

[5]许顺生,金属X射线学,上海科技出版社, 1962,190.

[6] Guinier, A. \& Fournet, G., Small Angle Scattering of X-rays Wiley. New York. 1955, $157,115$.

[ 7] Meng, C. (孟昭富), Wang, Y. (王爆明), J. Non-cryst. Solids, 81(1986), 397-401.

[8] Delaglio, F. et al., J. Catal., 99(1986), 383-390.

[9] Scott, M. G., J. Mat. Sci., 13(1978), 291. 